\title{
The Impact of Treatment Noncompliance on Mortality in People With Type 2 Diabetes
}

CRAig J. Currie, PhD ${ }^{1}$ Mark PeYrot, PHD ${ }^{2,3}$ Christopher Ll. Morgan, MSC ${ }^{4}$ Chris D. POOLE, PHD ${ }^{4}$
SARA Jenkins-Jones, MSC ${ }^{4}$ RICHARD R. RUBIN, PHD ${ }^{3}$ CHRISTOPHER M. BURTON, MD, PHD ${ }^{5}$ MARC Evans, MD ${ }^{6}$

OBJECTIVE-To assess the association of compliance with treatment (medication and clinic appointments) and all-cause mortality in people with insulin-treated type 2 diabetes.

RESEARCH DESICN AND METHODS - Data were extracted from U.K. general practice records and included patients $(N=15,984)$ who had diagnostic codes indicative of type 2 diabetes or who had received a prescription for an oral antidiabetic agent and were treated with insulin. Records in the 30 months before the index date were inspected for clinical codes (recorded at consultation) indicating medication noncompliance or medical appointment nonattendance. Noncompliance was defined as missing more than one scheduled visit or having at least one provider code for not taking medications as prescribed. Relative survival postindex date was compared by determining progression to all-cause mortality using Cox proportional hazards models.

RESULTS - Those identified as clinic nonattenders were more likely to be smokers, younger, have higher $\mathrm{HbA}_{1 \mathrm{c}}$, and have more prior primary care contacts and greater morbidity $(P<$ $0.001)$. Those identified as medication noncompliers were more likely to be women $(P=$ $0.001)$, smokers $(P=0.014)$, and have higher $\mathrm{HbA}_{1 \mathrm{c}}$, more prior primary care contacts, and greater morbidity (all $P<0.001$ ). After adjustment for confounding factors, medication noncompliance (hazard ratio 1.579 [95\% CI 1.167-2.135]), clinic nonattendance of one or two missed appointments (1.163 [1.042-1.299]), and clinic nonattendance of greater than two missed appointments (1.605 [1.356-1.900]) were independent risk factors for all-cause mortality.

CONCLUSIONS - Medication noncompliance and clinic nonattendance, assessed during routine care by primary care physicians or their staff, were independently associated with increased all-cause mortality in patients with type 2 diabetes receiving insulin.

Diabetes Care 35:1279-1284, 2012

$\mathbf{T}$ ype 2 diabetes is associated with excess mortality (1), higher health care costs (2), and reduced quality of life (3). The clinical antecedents of diabetes-related complications include poor glucose control, hypertension, and dyslipidemia, which result in a requirement for a combination of preventative or remedial strategies. While treatment efficacy for blood pressure and cholesterol

levels has improved in recent years, glucose control has improved little (2). A recent study in the U.S. finds that only $13.2 \%$ of people with diabetes achieved target levels for all three risk factors (4).

Although the choice of treatment regimen and the specific glucose-control target at a population level remains contentious (5), it is clear that an individual's failure to use prescribed medications

From the ${ }^{1}$ Department of Medicine, School of Medicine, Cardiff University, Cardiff, Wales, U.K.; ${ }^{2}$ Loyola University Maryland, Baltimore, Maryland; the ${ }^{3}$ School of Medicine, Johns Hopkins University, Baltimore, Maryland; ${ }^{4}$ Global Epidemiology, Pharmatelligence, Cardiff, Wales, U.K.; ${ }^{5}$ Point of Care Medical Consulting, Copenhagen, Denmark; and the ${ }^{6}$ Department of Medicine, University Hospital of Wales, Cardiff, Wales, U.K.

Corresponding author: Craig J. Currie, currie@cardiff.ac.uk.

Received 5 July 2011 and accepted 19 February 2012

DOI: $10.2337 / \mathrm{dcll}-1277$

( 2012 by the American Diabetes Association. Readers may use this article as long as the work is properly cited, the use is educational and not for profit, and the work is not altered. See http://creativecommons.org/ licenses/by-nc-nd/3.0/ for details.

properly-in terms of adherence and/or persistence-also contributes to adverse clinical outcomes. On average, half the patients prescribed medications for modifiable risk factors common to people with diabetes discontinue therapy within 12 months of treatment initiation (6). Poorly compliant patients miss scheduled appointments for diabetes-related monitoring and treatment (7), and this is associated with poor glucose control $(7,8)$.

Insulin is widely recognized as the most efficacious treatment for patients with type 2 diabetes (9), but successful treatment additionally requires regular blood glucose monitoring and insulin titration (10). The effects of treatment noncompliance in this patient population, therefore, may be expected to have a more dramatic impact on clinical outcomes.

The purpose of this study was to determine if poor medication compliance and/or nonattendance at scheduled medical appointments, as assessed by primary care providers or their staff during routine care, was associated with all-cause mortality in people with type 2 diabetes receiving insulin.

\section{RESEARCH DESIGN AND METHODS}

\section{Data source}

Data were extracted from The Health Improvement Network (THIN) database (11), a longitudinal, anonymized research database derived from $>350$ primary care practices in the U.K. The THIN database presently contains records for $>8$ million patients, of whom $\sim 4$ million are actively registered and can be followed prospectively (12). Available data include patient demographics, medical history (including diagnoses and health contacts), biochemistry and microbiology test results, and pharmaceutical prescriptions.

\section{Read codes}

Read codes are standardized codes used to record clinical data on computerized systems in primary care in the U.K. (13) and are routinely used to evaluate and 
reward practices' performance in relation to national treatment targets according to the Quality Outcomes Framework (14). These Read codes are cross-mapped to ICD-9 and ICD-10 (for diseases); and Office of Population, Censuses, and Surveys Classification of Surgical Operations and Procedures (OPCS)-4 (for procedures); and the British National Formulary (for medications). There are also many additional codes for data items, such as family history, occupation, adverse events, and social factors, as well as for medication compliance and appointment attendance. In most cases, the physician (or nurse for nurse-led consultations) recorded the codes at the time of the consultation. Administrative staff sometimes recorded other data, for example, data transcribed from letters from hospital consultants. The physician or nurse assessed medication noncompliance at the time of consultation based on assessment of the patient. This is a subjective measure based primarily on the physician's assessment at consultation (or transcribed from letters from hospital consultants). Contact nonattendance was recorded when the patient failed to attend a scheduled appointment at the primary care practice or information was fed back (via letter) that a patient had not attended a hospital appointment. This is an objective measure, though its application might not be uniform or systematic.

The diagnostic information in the THIN database is recorded using the Read code vocabulary.

\section{Patient selection and coding of diabetes type}

Patients were selected for the study if they had Read codes exclusively and explicitly indicative of type 2 diabetes. Where no specific type of diabetes was recorded or where, in a small number of instances, the patient history contained conflicting type 1 and type 2 codes, patients were classified as having type 2 diabetes if they had at least one record with a Read code indicative of type 2 diabetes and at least one prescription for an oral antidiabetic agent or if they had been prescribed two different classes of oral antidiabetic medication. Patients were excluded if they were aged $<35$ years at presentation and initiated on insulin with no prescriptions for any oral antidiabetic agents. Patients with secondary diabetes were also excluded.

Patients were required to have at least 36 months of care and follow-up before the mortality observation period, including at least 6 months of observation prior to their initiation of insulin therapy (a wash-in period), and at least 30 months of treatment with insulin during which their compliance could be assessed. Mortality was assessed from the end of the 36-month assessment period, which was defined as the index date. Patients with an index date between January 2000 and November 2009 were extracted for analysis, resulting in a study population of 15,984 . All patients were observed until death or data censorship (median [IQR] observation period $=3.16$ years [1.50-5.37]).

\section{Classification of treatment noncompliance}

In each cohort, patients were observed for an initial 30-month period during which their treatment noncompliance was determined. Specific Read codes within the dataset were used to characterize patients with respect to medication noncompliance and nonattendance at a primary care or hospital scheduled contact. Medication noncompliance was defined as noncompliance to any prescribed medication, including but not limited to diabetes medication. An episode of medication noncompliance or contact nonattendance was defined for each unique Read code occurring on each single day. Medication compliance was defined as a binary variable (no noncompliance Read codes vs. at least one noncompliance Read code). Contact attendance was classified as no nonattendance and two categories of noncompliance (from one to two missed appointments and greater than two missed appointments).

The use of the term noncompliance as opposed to nonadherence is intentional and reflects the nomenclature present in the Read codes.

\section{Statistical analysis}

Index date characteristics of compliance groups were compared using univariate statistics (ANOVA for continuous variables and $\chi^{2}$ for categorical variables). Relative survival in terms of the hazard of death for treatment compliant and noncompliant patients was assessed by determining the adjusted progression to all-cause mortality and evaluated with Cox proportional hazards models with forward manual inclusion (threshold statistical significance was $P \leq 0.05$ ) using SPSS version 18. The Cox proportional hazards model could include the following covariates assessed at index date (all were available and tested for inclusion in the model): age, sex, clinical values (BMI, $\mathrm{HbA}_{\mathrm{lc}}$, cholesterol, and blood pressure), smoking status, and morbidity, which was represented by binary variables for history of large vessel disease, renal disease, and visual disorders and by the Charlson Index (13). Also eligible for inclusion was a continuous variable quantifying the total number of primary care contacts in the year before index date. Ethnicity and social status could not be included because this information was not recorded. The survival model also included interaction terms to determine whether medication noncompliance and contact nonattendance had nonadditive associations with mortality.

Hazard ratios (HRs) are given with 95\% CIs. Case subjects with missing data were excluded from the survival model, resulting in an analytic sample of 13,401 patients.

\section{RESULTS}

Patient characteristics at index date The number of missed appointments (as indicated by the number of times that a visit nonattendance Read code was used) ranged from 0 to 39 in the entire cohort. The number of patients missing at least one scheduled appointment during the assessment period was 6,227 (39.0\%), of whom 4,346 missed from one to two appointments and 1,881 missed more than two appointments. The number of patients identified as noncompliant with medication was 705 (4.4\%). Of those patients missing one or more scheduled appointments, 423 (2.6\% of the entire cohort and $6.8 \%$ of those who missed more than one appointment) also were identified as noncompliant with medication. Appointment nonattendance was higher among those who were noncompliant with medication than those who were compliant with medication (odds ratio 2.45 [95\% CI 2.099-2.857]).

Table 1 shows the univariate associations between index date patient characteristics and both forms of treatment noncompliance. Compared with clinic attenders, clinic nonattenders were more likely to be smokers and younger and to have higher $\mathrm{HbA}_{\mathrm{lc}}$, more prior primary care contacts, and higher Charlson morbidity scores (all $P<0.001$ ). Compared with medication compliers, medication noncompliers were more likely to be women $(P=0.001)$ and smokers $(P=$ $0.014)$ and to have higher $\operatorname{HbA}_{1 \mathrm{c}}(P<$ $0.001)$, more prior primary care contacts $(P<0.001)$, and higher Charlson morbidity scores $(P<0.001)$. 
Table 1-Index date characteristics according to diagnosis of medication noncompliance and clinic nonattendance

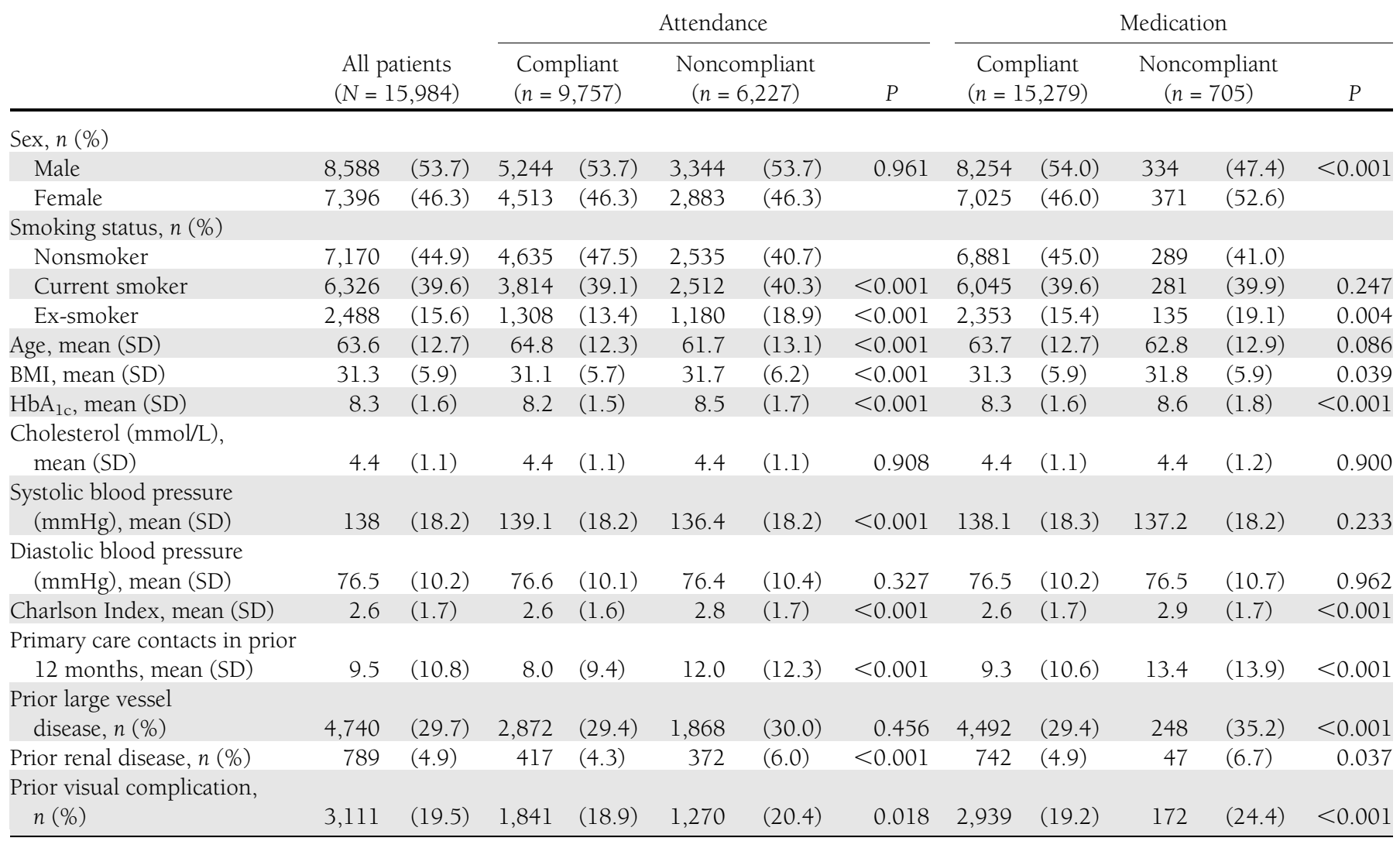

\section{Mortality}

The crude, unadjusted mortality rates were greater for those patients who were noncompliant for medication or clinic attendance (Table 2). Findings from the Cox regression analyses are presented in Table 3. After adjustment for confounding factors, the diagnosis of medication noncompliance increased the hazard for mortality significantly (HR 1.58 [95\% CI 1.172.14]). Clinic nonattendance was associated with increased mortality hazard of 1.16 (1.04-1.30) for those missing one to two appointments and 1.61 (1.36-1.90) for those missing two or more appointments. There was a significant interaction between nonattendance and medication noncompliance. The interaction represents the fact that the group with both risk factors has a significantly lower risk than it would have if the effects were additive. That is, the group with both risk factors is not at significantly higher risk than that with only one risk factor, whereas it would be significantly higher if the main effects were additive. To specify the nature of this interaction, we conducted an ancillary analysis. Among those who were medication compliant, there was a significant $(P<$ $0.001)$ monotonic increase in mortality as nonattendance increased; among those who were medication noncompliant, there was no significant difference in mortality as nonattendance increased $(P=0.489)$.

A sensitivity analysis was performed for patients with a minimum of 1 month's survival; both indicators of treatment noncompliance remained significantly associated with mortality.

CONCLUSIONS - The principal finding of this study was that two measures of

Table 2-Number of death events, length of follow-up periods, crude event rates, and crude relative risks

\begin{tabular}{|c|c|c|c|c|c|c|}
\hline Compliance group & $n$ & Deaths & $\begin{array}{c}\text { Follow-up } \\
\text { (patient-years) }\end{array}$ & $\begin{array}{c}\text { Mortality rate (per } 1,000 \\
\text { patient-years) }\end{array}$ & $\begin{array}{l}\text { Relative risk (95\% CI) } \\
\text { (reference is compliant) }\end{array}$ & $P$ \\
\hline Compliant & 9,475 & 1,546 & 38,009 & 40.7 & & \\
\hline $\begin{array}{l}\text { Diagnosis of medication } \\
\text { noncompliance* }\end{array}$ & 705 & 99 & 1,870 & 52.9 & $1.302(1.057-1.588)$ & 0.014 \\
\hline Clinic nonattendance** & 6,227 & 821 & 18,532 & 44.3 & $1.089(1.000-1.169)$ & 0.049 \\
\hline 1-2 missed appointments** & 4,346 & 596 & 13,657 & 43.6 & $1.073(0.976-1.179)$ & 0.1446 \\
\hline$>2$ missed appointments** & 1,881 & 225 & 4,875 & 46.2 & $1.135(0.985-1.303)$ & 0.080 \\
\hline
\end{tabular}

*Includes patients in clinic nonattendance rows. ${ }^{* *}$ Includes patients in medication noncompliance row. 
Table 3-Multivariate Cox regression model of all-cause mortality $(\mathrm{N}=13,401)$ *

\begin{tabular}{|c|c|c|}
\hline & $\operatorname{HR}(95 \% \mathrm{CI})$ & $P$ \\
\hline Age (years) & $1.085(1.079-1.090)$ & $<0.001$ \\
\hline Smoking status (reference is nonsmoker) & & $<0.001$ \\
\hline Ex-smoker & $1.139(1.028-1.262)$ & 0.013 \\
\hline Current smoker & $1.470(1.270-1.701)$ & $<0.001$ \\
\hline Female (reference is male) & $0.805(0.731-0.886)$ & $<0.001$ \\
\hline Systolic blood pressure (mmHg) & $0.995(0.992-0.997)$ & $<0.001$ \\
\hline $\mathrm{HbA}_{\mathrm{lc}}$ & $1.047(1.015-1.080)$ & 0.004 \\
\hline Primary care contacts in prior 12 months & $1.011(1.008-1.014)$ & $<0.001$ \\
\hline Renal disease & $1.703(1.416-2.048)$ & $<0.001$ \\
\hline Medication noncompliance & $1.579(1.167-2.135)$ & 0.003 \\
\hline Clinic nonattendance & & $<0.001$ \\
\hline 1-2 missed appointments & $1.163(1.042-1.299)$ & 0.007 \\
\hline$>2$ missed appointments & $1.605(1.356-1.900)$ & $<0.001$ \\
\hline Interaction terms & & 0.017 \\
\hline $\begin{array}{l}\text { Medication noncompliance and 1-2 } \\
\text { missed appointments }\end{array}$ & $0.685(0.419-1.120)$ & 0.132 \\
\hline $\begin{array}{l}\text { Medication noncompliance and }>2 \\
\text { missed appointments }\end{array}$ & $0.397(0.205-0.766)$ & 0.006 \\
\hline
\end{tabular}

*All estimates adjusted for known risk factors for mortality in patients with type 2 diabetes reaching threshold significance $(P<0.05)$ in the multivariate model.

treatment noncompliance made by primary care physicians or their staff as a part of routine clinical care (i.e., a primary care physician's diagnosis of medication noncompliance and visit nonattendance) were independently associated with increased mortality in insulin-treated patients with type 2 diabetes, after controlling for clinical factors known to be associated with mortality. Although patients defined as noncompliant by either metric were more likely to smoke and have higher $\mathrm{BMI}, \mathrm{HbA}_{1 \mathrm{c}}$, and morbidity at index date, the association of treatment noncompliance with subsequent mortality was independent of these risk factors. Furthermore, there was a dose-response relationship between level of missed appointments and mortality risk. Finally, there was a significant interaction between forms of treatment noncompliance involving a negative synergy in which the effect of one form of treatment noncompliance was reduced in the presence of the other form of noncompliance.

These findings can be compared with those of prior studies. Karter et al. (7) reported that missing scheduled medical appointments was associated with higher $\mathrm{HbA}_{\mathrm{lc}}$ levels - a predictor of mortality - and with poor oral medication refill adherence. Rhee et al. (8) found that appointment keeping and medication adherence were each independently associated with $\mathrm{HbA}_{1 \mathrm{c}}$ levels and that those with both risk factors had the highest $\mathrm{HbA}_{1 \mathrm{c}}$ levels.
As far as the authors are aware, however, our study is the first to demonstrate independent, prospective associations of medication noncompliance and clinic nonattendance with mortality. Although it was not possible to examine potential mechanisms accounting for these associations, it is likely that treatment noncompliance prior to index date resulted in increases in the number and severity of complications over time and, in turn, increased mortality.

While the associations of mortality with medication noncompliance and clinic nonattendance are independent, they are not additive. We cannot offer a definitive explanation of this lack of additivity, but it appears that patients who take their medication as prescribed benefit from increased provider supervision (fewer missed visits) while those who do not take their medication as prescribed do not benefit from increased supervision, perhaps because they are noncompliant with other (nonmedication) provider directives. Further research is needed to confirm these findings and to examine possible explanations.

Although there has been little research demonstrating that assessment of treatment noncompliance by primary care physicians can identify patients who are at increased mortality risk, one study reports that primary care physicians' assessment of patient compliance predicted mortality in patients with type 2 diabetes (15). In that study, compliance was assessed as part of a research protocol that asked for an assessment outside the routine delivery of care, based on training provided during the study. Our research goes beyond this earlier study to show that assessments made during routine clinical care by unselected primary care providers without special training in compliance assessment are also able to identify diabetic patients at significantly increased risk of mortality. These assessments of noncompliance predicted mortality even when controlling for initial levels of several physiological factors by which compliance might affect mortality (for example, comorbidity and metabolic control).

There are also very limited data available investigating the reasons underlying patient nonattendance at medical appointments, particularly in the field of diabetes. In a study of nonattendance at a family practice in Mexico City among patients with type 2 diabetes or hypertension or attending antenatal care, there were approximately equal numbers of patients either forgetting appointments or having experienced administrative problems (16), suggesting that organizational procedures may be significant contributing factors. In another study (17), providing patients with sufficient information reduced new patient nonattendance from 15 to almost $1 \%$ in a specialist diabetes clinic in the U.K. In the current study, we report considerably higher nonattendance rates of 39\% during a 30-month period. Possible reasons for the discrepancy might be differences in patient-perceived value between attending a specialist versus a primary care clinic appointment or between initial versus follow-up clinic appointments. Whatever the cause, having $\sim 40 \%$ of patients miss at least one appointment represents both a substantial waste of health care resources and a risk for poor health outcomes.

This study has several strengths. These include the large study samples of patients with type 2 diabetes and providers who treat and diagnose these patients. The study included a wash-in period to assess patient treatment history and a lengthy period during which treatment noncompliance could be assessed. The observed findings with respect to patient age, BMI, $\mathrm{HbA}_{\mathrm{lc}}$, and current smoking across the compliance categories are also consistent with previous reports (18-20). Finally, the study was able to assess the ultimate patient-relevant outcome: mortality. 
This study also has limitations. The Read codes used to identify patients who were medication noncompliant are subjective. Other methods, such as biological testing of fluid or tissue samples, and more practical methods, such as pill counts, more accurately determine whether patients are complying with their recommended medication. In a similar manner, while nonattendance may be considered objective, it is not clear whether all primary care practices or individual physicians or their staff record nonattendance with the same thoroughness.

In addition, while we believe that our general conclusions are valid, any interpretation needs to consider potential pitfalls common in this type of epidemiological study, such as unmeasured confounders. For example, ethnicity and social status could not be included in this analysis, and it is plausible that these factors are common to those patients who are both noncompliant and have reduced survival. However, the analyses controlled for a large number of other demographic and medical factors that might account for the relationship between treatment noncompliance and mortality. In the current study, it was not possible to perform the analysis with compliance as a time-varying factor because of the way in which the data were recorded in the THIN database. Compliance may change over time; however, the effect of patients switching between compliance and noncompliance would be expected to make both groups more similar and, thus, the reported HR is likely to be a conservative estimate. Finally, it was not possible to examine whether treatment noncompliance was associated with deterioration in glucose control and increased morbidity over time and whether this accounted for the prospective association between treatment noncompliance and mortality.

Treatment noncompliance is being increasingly recognized as one of the major limitations to improving health care outcomes, and a greater understanding of the relationships between noncompliance and treatment practices will be important in guiding future care practices and medication development. Polypharmacy and increased frequency and complexity of medication administration are known to be associated with higher rates of medication noncompliance $(21,22)$.

The diagnostic, prescriptive, and procedural accuracy of Read codes in the U.K. has been determined previously (23); however, the practices through which health care professionals assign the codes defining medication noncompliance are yet to be determined. Diagnoses of noncompliance may be used to exclude patients from the pay-for-performance assessment used in the U.K. (14). The threat of an audit for practices reporting higher rates of patient noncompliance may mean that only those patients where there is a significant, ongoing, and noticeable problem are diagnosed as such. It is highly likely, therefore, that many more patients than those reported in this study were noncompliant with their medications. It is arguable that if this diagnosis were disengaged from financial remuneration, we would be better able to evaluate the impact of noncompliance on treatment targets and health care outcomes.

The approach used in this study appears to be easily adaptable for use in general clinical practice. Patients could be flagged for follow-up when they have missed one or more visits or are thought by the primary care physician to be noncompliant with their medication regimen (the compliance indicators associated with increased mortality in the current study). With greater attention to coding treatment noncompliance, physicians might be able to identify more patients who have elevated mortality risk and intervene to reduce noncompliance. Moreover, if future research shows a similar association between treatment noncompliance and mortality in nondiabetic populations, this approach may have broader applicability.

With currently available treatments, medication noncompliance and clinic nonattendance were associated with increased all-cause mortality in patients with insulin-treated type 2 diabetes. These associations were independent of each other and the mortality risk associated with suboptimal control of routinely targeted metabolic control parameters. Understanding and addressing factors that promote patient noncompliance will be important if we are to improve the life expectancy of patients with insulin-treated type 2 diabetes.

Acknowledgments-This study was funded by an unconditional research grant from Novo Nordisk. C.J.C. has received consulting fees and/or research grants from Amylin, ARYx, Astellas, Boehringer Ingelheim, Bristol-Myers Squibb, Eisel, Ferring, GlaxoSmithKline, Ipsen, Eli Lilly, Medtronic, Merck, Pfizer, sanofiaventis, Takeda, and Wyeth. M.P. has received consulting fees and/or research grants from
Amylin, Animas, CPEX, Eli Lilly, Genentech, Healthy Interactions, MannKind, Medtronic, Novo Nordisk, and Patton Medical Devices. C.Ll.M., C.D.P., and S.J.-J. are employees of Pharmatelligence. R.R.R. has received consulting fees and/or research grants from Animas, Amylin, Eli Lilly, LifeScan, MannKind, Medingo, Medtronic MiniMed, Novo Nordisk, Patton Medical Devices, Roche, and sanofi-aventis. C.M.B. has received consulting fees from Novo Nordisk. M.E. has received consulting fees from Abbott, Allergan, Bristol-Myers Squibb, GlaxoSmithKline, Eli Lilly, Novartis, Novo Nordisk, Merck Sharp \& Dohme, Roche, sanofi-aventis, and Takeda. No other potential conflicts of interest relevant to this article were reported.

C.J.C. wrote the protocol, researched data, contributed to discussion, and wrote the manuscript. M.P. contributed to discussion and wrote the manuscript. C.Ll.M. researched data, contributed to discussion, and wrote the manuscript. C.D.P. researched data and contributed to discussion. S.J.-J. researched data. R.R.R. contributed to discussion and reviewed and edited the manuscript. C.M.B. wrote the protocol and provided editorial assistance. M.E. contributed to discussion and reviewed and edited the manuscript. C.J.C. is the guarantor of this work and, as such, had full access to all the data in the study and takes responsibility for the integrity of the data and the accuracy of the data analysis.

Parts of this study were presented in abstract form at the 47th Annual Meeting of the European Association for the Study of Diabetes, Lisbon, Portugal, 12-16 September 2011.

\section{References}

1. Roper NA, Bilous RW, Kelly WF, Unwin NC, Connolly VM. Excess mortality in a population with diabetes and the impact of material deprivation: longitudinal, population based study. BMJ 2001;322:1389-1393

2. Currie CJ, Gale EA, Poole CD. Estimation of primary care treatment costs and treatment efficacy for people with type 1 and type 2 diabetes in the United Kingdom from 1997 to 2007. Diabet Med 2010;27: 938-948

3. Manuel DG, Schultz SE. Health-related quality of life and health-adjusted life expectancy of people with diabetes in Ontario, Canada, 1996-1997. Diabetes Care 2004;27:407-414

4. Ong KL, Cheung BM, Wong LY, Wat NM, Tan KC, Lam KS. Prevalence, treatment, and control of diagnosed diabetes in the U.S. National Health and Nutrition Examination Survey 1999-2004. Ann Epidemiol 2008;18:222-229

5. Yudkin JS, Richter B, Gale EA. Intensified glucose lowering in type 2 diabetes: time for a reappraisal. Diabetologia 2010;53: 2079-2085

6. Zafar A, Davies M, Azhar A, Khunti K. Clinical inertia in management of T2DM. Prim Care Diabetes 2010;4:203-207 
7. Karter AJ, Parker MM, Moffet HH, et al Missed appointments and poor glycemic control: an opportunity to identify highrisk diabetic patients. Med Care 2004;42: 110-115

8. Rhee MK, Slocum W, Ziemer DC, et al. Patient adherence improves glycemic control. Diabetes Educ 2005;31:240 250

9. Nathan DM, Buse JB, Davidson MB, et al.; American Diabetes Association; European Association for the Study of Diabetes. Medical management of hyperglycemia in type 2 diabetes: a consensus algorithm for the initiation and adjustment of therapy: a consensus statement of the American Diabetes Association and the European Association for the Study of Diabetes. Diabetes Care 2009;32:193-203

10. Karter AJ, Ackerson LM, Darbinian JA, et al. Self-monitoring of blood glucose levels and glycemic control: the Northern California Kaiser Permanente Diabetes Registry. Am J Med 2001;111:1-9

11. The Health Improvement Network [article online], 2010. Cegedim Strategic Data/ EPIC. Available from http://csdmruk. cegedim.com/our-data/our-data.html. Accessed 9 January 2011
12. Maguire A, Blak BT, Thompson M. The importance of defining periods of complete mortality reporting for research using automated data from primary care. Pharmacoepidemiol Drug Saf 2009;18: 76-83

13. National Health Service. Read codes [article online], 2011. Available from http:// www.connectingforhealth.nhs.uk/ systemsandservices/data/readcodes. Accessed 9 May 2011

14. Quality and Outcomes Framework guidance for GMS contract 2009/10, 2009. Available from http://www.bma.org.uk/ images/qof0309_tcm41-184025.pdf. Accessed 9 May 2011

15. Rothenbacher D, Rüter G, Brenner $H$. Prognostic value of physicians' assessment of compliance regarding all-cause mortality in patients with type 2 diabetes: primary care follow-up study. BMC Fam Pract 2006; 7:42

16. Medécigo-Micete C, Constantino-Casas $\mathrm{P}$, Rodríguez-Pacheco JL. Causes for failure of the medical appointment program in a family medicine clinic. Rev Med Inst Mex Seguro Soc 2007;45:213-217 [in Spanish]

17. Hardy KJ, O'Brien SV, Furlong NJ. Information given to patients before appointments and its effect on non-attendance rate. BMJ 2001:323:1298-1300

18. Donnelly LA, Morris AD, Evans JM; DARTS/MEMO collaboration. Adherence to insulin and its association with glycaemic control in patients with type 2 diabetes. QJM 2007;100:345-350

19. Yang Y, Thumula V, Pace PF, Banahan BF 3rd, Wilkin NE, Lobb WB. Predictors of medication nonadherence among patients with diabetes in Medicare Part D programs: a retrospective cohort study. Clin Ther 2009; 31:2178-2188; discussion 2150-2151

20. Yurgin NR, Boye KS, Dilla T, Suriñach NL, Llach XB. Physician and patient management of type 2 diabetes and factors related to glycemic control in Spain. Patient Prefer Adherence 2008;2:87-95

21. Peyrot M, Rubin RR, Kruger DF, Travis LB. Correlates of insulin injection omission. Diabetes Care 2010;33:240-245

22. Rubin RR. Adherence to pharmacologic therapy in patients with type 2 diabetes mellitus. Am J Med 2005;118(Suppl. 5A): 27S-34S

23. Hassey A, Gerrett D, Wilson A. A survey of validity and utility of electronic patient records in a general practice. BMJ 2001; 322:1401-1405 